Homology, Homotopy and Applications, vol.17(2), 2015, pp.137-160

\title{
OPERATIONS ON POLYHEDRAL PRODUCTS AND A NEW TOPOLOGICAL CONSTRUCTION OF INFINITE FAMILIES OF TORIC MANIFOLDS
}

\author{
A. BAHRI, M. BENDERSKY, F. R. COHEN AND S. GITLER
}

(communicated by Donald M. Davis)

\begin{abstract}
A combinatorial construction is used to analyze the properties of polyhedral products [1] and generalized moment-angle complexes with respect to certain operations on $\mathrm{CW}$ pairs including exponentiation. This allows for the construction of infinite families of toric manifolds, associated to a given one, in a way that simplifies the combinatorial input and, consequently, the presentation of the cohomology rings. The new input is the interaction of a purely combinatorial construction with natural associated geometric constructions related to polyhedral products and toric manifolds. Applications of the methods and results developed here have appeared in $[24,25,15,18,10,23]$, and $[19]$.
\end{abstract}

This paper is dedicated to the memory of Samuel Gitler.

\section{Introduction}

The polyhedral product $Z(K ;(\underline{X}, \underline{A}))$ is a $\mathrm{CW}$-complex valued functor of two variables: the first, an abstract simplicial complex $K$ on $m$ vertices and the second, a family of (based) CW pairs

$$
(\underline{X}, \underline{A})=\left\{\left(X_{1}, A_{1}\right),\left(X_{2}, A_{2}\right), \ldots,\left(X_{m}, A_{m}\right)\right\} .
$$

It is defined as a union of products inside $\prod_{i=1}^{m} X_{i}$ each parameterized by a simplex in the simplicial complex $K$.

Polyhedral products generalize the spaces called moment-angle complexes which were developed first by Buchstaber and Panov in [7] and correspond to the case $\left(X_{i}, A_{i}\right)=\left(D^{2}, S^{1}\right), i=1,2, \ldots, m$. At the core of the subject of toric topology, which began as a topological approach to toric geometry, are three families of spaces:

Received August 13, 2014, revised January 5, 2015, January 17, 2015; published on November 11, 2015.

2010 Mathematics Subject Classification: Primary: 52B11, 55N10, 14M25, 55U10, 13F55. Secondary: $14 \mathrm{~F} 45,55 \mathrm{~T} 10$.

Key words and phrases: polyhedral product, moment-angle complex, moment-angle manifold, quasitoric manifold, toric manifold, quasitoric manifold, smooth toric variety, non-singular toric variety, fan, simplicial wedge, join.

Article available at http://dx.doi.org/10.4310/HHA.2015.v17.n2.a8

Copyright (C) 2015, International Press. Permission to copy for private use granted. 
1. polyhedral products and moment-angle manifolds,

2. Davis-Januszkiewicz spaces, and

3. toric manifolds and real toric manifolds.

This paper is devoted to an analysis of the properties of these spaces with respect to an operation

$$
J: K \longrightarrow K(J)
$$

from abstract simplicial complexes with $m$ vertices to abstract simplicial complexes, which is determined by a sequence of positive integers $J=\left(j_{1}, j_{2}, \ldots, j_{m}\right)$. Here, the simplicial complex $K(J)$ has $d(J)=j_{1}+j_{2}+\cdots+j_{m}$ vertices. Several applications to toric topology are discussed in detail.

By abuse of notation, the symbol $Z(K(J) ;(\underline{X}, \underline{A}))$ is used to denote the polyhedral product determined by the simplicial complex $K(J)$ and the family of pairs obtained from $(\underline{X}, \underline{A})$ by repeating each $\left(X_{i}, A_{i}\right), j_{i}$ times in sequence. In Section 7 , another operation $\bar{J}$ is defined on families of CW-pairs yielding an identity of polyhedral products

$$
Z(K(J) ;(\underline{X}, \underline{A}))=Z(K ; \bar{J}(\underline{X}, \underline{A})) .
$$

This result has consequences for the case

$$
(\underline{X}, \underline{A})=\left(\underline{D}^{2 J}, \underline{S}^{2 J-1}\right)=\left\{\left(D^{2 j_{i}}, S^{2 j_{i}-1}\right)\right\}_{i=1}^{m},
$$

corresponding to the polyhedral products that are called now generalized momentangle complexes. The central result here is the following.

Theorem 7.5. There is an action of $T^{m}$ on polyhedral products $Z\left(K ;\left(\underline{D}^{2 J}, \underline{S}^{2 J-1}\right)\right)$ and $Z\left(K(J) ;\left(D^{2}, S^{1}\right)\right)$, with respect to which they are equivariantly homeomorphic.

When combined with Theorem 1.8 of [3], this theorem yields an immediate corollary.

Corollary 7.6. The spaces $Z\left(K ;\left(D^{2}, S^{1}\right)\right)$ and $Z\left(K(J) ;\left(D^{2}, S^{1}\right)\right)$ have isomorphic ungraded cohomology rings.

Another application links the spaces $\left(\underline{D}^{2 J}, \underline{S}^{2 J-1}\right)$ to the study of toric manifolds in a new way.

For the much studied case $\left(X_{i}, A_{i}\right)=\left(D^{1}, S^{0}\right), i=1,2, \ldots, m$, it follows from the results in Section 7 that every moment-angle complex $Z\left(K ;\left(D^{2}, S^{1}\right)\right)$ can be realized as $Z\left(K(J) ;\left(D^{1}, S^{0}\right)\right)$ for $J=(2,2, \ldots, 2)$. Therefore, in a certain sense, the "real" moment-angle complex is the more basic object. In the context of toric manifolds over a simple polytope $P^{n}$, with simplicial complex $K$ dual to the boundary $\partial P^{n}$, the spaces $Z\left(\partial P^{n} ;\left(D^{2}, S^{1}\right)\right)$ and $Z\left(\partial P^{n} ;\left(D^{1}, S^{0}\right)\right)$, which in this case are differentiable manifolds, were introduced by Davis and Januszkiewicz in [12]. They used the latter to construct spaces known as small covers, the subject of considerable current investigation.

The fundamental construction of Davis and Januszkiewicz [12, Section 1.5] realizes all toric manifolds $M^{2 n}$ and, in particular, all smooth projective toric varieties. From this construction it follows that $M^{2 n}$ can be described as the quotient of an $(m+$ $n$ )-dimensional moment-angle complex by the free action of a certain real $(m-n)$ dimensional torus $T^{m-n} \subset T^{m}$. This subtorus is specified usually by a characteristic map $\lambda$. 
Beginning with a toric manifold $M^{2 n}$, its associated simple polytope $P^{n}$ having $m$ facets and characteristic map $\lambda$, an infinite family of new toric manifolds $M(J)$ is constructed, one for each sequence of positive integers $J=\left(j_{1}, j_{2}, \ldots, j_{m}\right)$. The manifolds $M(J)$ are determined by a new polytope $P(J)$ and a new characteristic map $\lambda(J)$. The outcome here (Theorem 4.2) is that the integral cohomology ring of $M(J)$ is described completely in terms of the original map $\lambda$ and the original polytope $P^{n}$. The manifolds $M(J)$ are a substantial new concrete class of toric manifolds that come equipped with a complete fan (where appropriate), combinatorial, and topological information. These spaces are a new, systematic infinite family of toric manifolds which have tractable as well as natural properties. Furthermore, these constructions arise from an operation defined on a category of finite simplicial complexes sending $K$ to $K(J)$ and so provide a natural construction.

In Section 9, the construction and properties of these manifolds $M(J)$ are analyzed in the context of generalized moment-angle complexes. As above, to the polytope $P^{n}$ is associated the simplicial complex $K$ dual to $\partial P^{n}$ and a generalized moment-angle complex $Z\left(K ;\left(\underline{D}^{2 J}, \underline{S}^{2 J-1}\right)\right)$, and to $P(J)$, which has boundary dual to $K(J)$, is associated the moment-angle complex $Z\left(K(J) ;\left(D^{2}, S^{1}\right)\right)$. Theorem 7.5 connects the construction $K(J)$ to the study of toric manifolds. In describing the new manifolds $M(J)$, diffeomorphisms (Theorems 7.5 and 9.2)

$$
Z\left(K(J) ;\left(D^{2}, S^{1}\right)\right) / T^{m-n} \longrightarrow M(J) \longleftarrow Z\left(K ;\left(\underline{D}^{2 J}, \underline{S}^{2 J-1}\right)\right) / T^{m-n}
$$

mirror geometrically the reduction in combinatorial complexity from the characteristic pair $(\lambda(J), P(J))$ to the pair $\left(\lambda, P^{2 n}\right)$. Significant in the theory of toric manifolds is the role played by the Davis-Januszkiewicz spaces. These are homotopy equivalent to polyhedral products of the form $Z\left(K ;\left(\mathbb{C} P^{\infty}, *\right)\right)$. Key in the theory that is developed here are related spaces, denoted by $Z\left(K ;\left(\underline{\mathbb{C}}^{\infty}, \mathbb{\mathbb { C } P}{ }^{J-1}\right)\right)$. They substitute for the usual Davis-Januszkiewicz spaces $Z\left(K(J) ;\left(\mathbb{C} P^{\infty}, *\right)\right)$. Their properties and relationship to the manifolds $M(J)$ are discussed in Theorems 10.5 and 11.1. In contrast to the cohomology of the Davis-Januszkiewicz spaces, these spaces have integral cohomology rings that are monomial ideal rings, but the monomials are not necessarily square-free. The construction of the toric manifolds $M(J)$ leads to the idea of "nests" of toric manifolds, which is discussed in the final section.

Remark. Unless indicated otherwise, all cohomology rings throughout are taken with integral coefficients.

The intersections of certain quadrics are known to be diffeomorphic to momentangle manifolds $[\mathbf{6}, \mathbf{1 7}]$, and, implicitly, in [7, Construction 3.1.8]. After a first draft of this article was written in 2008, the authors learned of the work of S. Lopez de Medrano on the intersections of quadrics $[\mathbf{2 1}, \mathbf{2 0}]$. Results in $[\mathbf{2 0}]$ depend on the consequences of a doubling of variables and a duplication of coefficients in the defining equations; this translates into an instance of the general construction given here.

The simplicial wedge construction, in the context of toric varieties, has appeared in the work of G. Ewald [16]. The ideas presented are distinguished from those of [16] by virtue of the following:

1. Not all the manifolds $M(J)$ discussed here are non-singular toric varieties.

2. The combinatorial constructions are analyzed in the context of polyhedral products, culminating in Theorem 7.2. 
3. A theory is constructed for the manifolds $M(J)$ that realizes them as quotients of generalized moment-angle complexes and parallels constructions developed for toric manifolds by Davis and Januszkiewicz $[\mathbf{1 2}]$ and Buschstaber and Panov $[\mathbf{9}]$.

Moreover, it is shown in the preprint [5] that the manifolds $M(J)$ can be described directly by a generalization of Davis and Januszkiewicz's original construction.

As is mentioned in the abstract, several applications of the constructions presented here have appeared both in the literature and in preprint form.

\section{Acknowledgments}

The authors are grateful to Carl Lee and Taras Panov for many interesting and helpful conversations, in particular for bringing to the authors' attention the fact that the simplicial complex $K\left(v_{i}\right)$, described by (1), is dual to the boundary of a simple polytope if $K$ is. Carl Lee provided the authors with an explicit proof of this theorem before the discovery of the result in the work of Provan and Billera [22]. Originally, the authors had arrived at $K\left(v_{i}\right)$ from the point of view of the generalized momentangle complexes that are discussed in Section 6. The authors thank Peter Landweber for his careful reading of the manuscript and for his many valuable suggestions. The authors are grateful to the referee for detecting various errors and for suggestions that have improved the paper.

The first author was supported in part by the award of a Rider University Research Leave and Research Fellowship and grant number 210386 from the Simons Foundation. The third author thanks the Department of Mathematics at the University of Pennsylvania for partial support as well as for a fertile environment during the Fall of 2010. He was partially supported also by DARPA grant number 2006-06918-01. The fourth author received support from CONACYT, Mexico.

\section{A construction on simplicial complexes}

Let $K$ be a simplicial complex of dimension $n-1$ on vertices $\left\{v_{1}, v_{2}, \ldots, v_{m}\right\}$, and let $J=\left(j_{1}, j_{2}, \ldots, j_{m}\right)$ be a sequence of positive integers.

Definition 2.1. A minimal non-face of a simplicial complex $K$ is a sequence of vertices of $K$ that is not a simplex of $K$ but any proper subset is a simplex of $K$. Let $K$ be as above. Denote by $K(J)$ the simplicial complex on $j_{1}+j_{2}+\cdots+j_{m}$ new vertices, labelled

$$
v_{11}, v_{12}, \ldots, v_{1 j_{1}}, v_{21}, v_{22}, \ldots, v_{2 j_{2}}, \ldots, v_{m 1}, v_{m 2}, \ldots, v_{m j_{m}},
$$

with the property that

$$
\left\{v_{i_{1} 1}, v_{i_{1} 2}, \ldots, v_{i_{1} j_{i_{1}}}, v_{i_{2} 1}, v_{i_{2} 2}, \ldots, v_{i_{2} j_{i_{2}}}, \ldots, v_{i_{k} 1}, v_{i_{k} 2}, \ldots, v_{i_{k} j_{i_{k}}}\right\}
$$

is a minimal non-face of $K(J)$ if and only if $\left\{v_{i_{1}}, v_{i_{2}}, \ldots, v_{i_{k}}\right\}$ is a minimal non-face of $K$. Moreover, all minimal non-faces of $K(J)$ have this form.

An alternative construction of the simplicial complex $K(J)$ will reveal the fact that $K(J)$ is dual to the boundary of $P(J)$ if $K$ is dual to $\partial P^{n}$. Recall that for $\sigma \in K$, 
the link of $\sigma$ in $K$ is the set

$$
\operatorname{link}_{K} \sigma:=\{\tau \in K: \sigma \cup \tau \in K, \sigma \cap \tau=\emptyset\} .
$$

The join of two simplicial complexes $K_{1}, K_{2}$ on disjoint vertex sets $S_{1}$ and $S_{2}$, respectively, is given by

$$
K_{1} * K_{2}:=\left\{\sigma \subset S_{1} \cup S_{2}: \sigma=\sigma_{1} \cup \sigma_{2}, \sigma_{1} \in K_{1}, \sigma_{2} \in K_{2}\right\} .
$$

Construction 1. As above, let $K$ be the simplicial complex on vertices $\left\{v_{1}, v_{2}, \ldots\right.$, $\left.v_{m}\right\}$. Choose a fixed vertex $v_{i}$ in $K$ and define a new simplicial complex $K\left(v_{i}\right)$ with $m+1$ vertices labelled $v_{1}, \ldots, v_{i-1}, v_{i 1}, v_{i 2}, v_{i+1}, \ldots, v_{m}$, by

$$
K\left(v_{i}\right):=\left\{v_{i 1}, v_{i 2}\right\} * \operatorname{link}_{K}\left\{v_{i}\right\} \cup\left\{\left\{v_{i 1}\right\},\left\{v_{i 2}\right\}\right\} *\left(K \backslash\left\{v_{i}\right\}\right) .
$$

Next, the vertices of $K\left(v_{i}\right)$, other than $v_{i 1}$ and $v_{i 2}$, are re-labelled by setting $v_{k}=v_{k 1}$ if $k \neq i$. Therefore, the new vertex set of $K\left(v_{i}\right)$ becomes

$$
S=\left\{v_{11}, \ldots, v_{(i-1) 1}, v_{i 1}, v_{i 2}, v_{(i+1) 1}, \ldots, v_{m 1}\right\} .
$$

Example 1. The easiest example is that of $K=\left\{v_{1}\right\},\left\{v_{2}\right\}$ two disjoint points. Here, $K\left(v_{1}\right)$ has three vertices $\left\{v_{11}, v_{12}, v_{21}\right\}, \operatorname{link}_{K}\left\{v_{1}\right\}=\varnothing$, and $K \backslash\left\{v_{1}\right\}=v_{2}$. So (1) becomes

$$
\left\{v_{11}, v_{12}\right\} * \varnothing \cup\left\{\left\{v_{11}\right\},\left\{v_{12}\right\}\right\} *\left\{v_{2}\right\}=\left\{v_{11}, v_{12}\right\} \cup\left(\left\{v_{11}, v_{21}\right\} \cup\left\{v_{12}, v_{21}\right\}\right),
$$

which is the boundary of a two-simplex.

In [22, p. 578], this construction is called the simplicial wedge of $K$ on $v$. Notice that if $\left\{v_{i_{1}}, v_{i_{2}}, \ldots, v_{i_{k}}\right\}$ is a minimal non-face of $K$ with $i_{j} \neq i$ for all $j$, then it remains a minimal non-face of $K\left(v_{i}\right)$. The simplex $\left\{v_{i 1}, v_{i 2}\right\}$ becomes part of a simplex

$$
\left\{v_{i_{1} 1}, v_{i_{2} 1}, \ldots, v_{i_{k} 1}, v_{i 1}, v_{i 2}, v_{i_{(k+1)} 1}, \ldots, v_{i_{s} 1}\right\} \in\left\{v_{i 1}, v_{i 2}\right\} * \operatorname{link}_{K}\left\{v_{i}\right\} \subseteq K\left(v_{i}\right)
$$

if and only if

$$
\left\{v_{i_{1}}, v_{i_{2}}, \ldots, v_{i_{k}}, v_{i}, v_{i_{k+1}}, \ldots, v_{i_{s}}\right\} \in K
$$

Hence, according to Definition 2.1, $K\left(v_{i}\right)=K(J)$, where

$$
J=(1,1, \ldots, 1,2,1, \ldots, 1)
$$

is the $m$-tuple with "2" appearing in the $i$ th coordinate. According to [22, p. 582], $K\left(v_{i}\right)$ is dual to the boundary of a simple polytope $P\left(v_{i}\right)$ of dimension $n+1$ with $m+1$ facets if $K$ is dual to the boundary of a simple polytope $P^{n}$ of dimension $n$ with $m$ facets. Beginning with $J=(1,1, \ldots, 1)$, Construction 1 may be iterated to produce $K(J)$ for any $J=\left(j_{1}, j_{2}, \ldots, j_{m}\right)$. The induction from $J=\left(j_{1}, j_{2}, \ldots, j_{m}\right)$ to the new sequence $J^{\prime}=\left(j_{1}, j_{2}, \ldots, j_{i-1}, j_{i}+1, j_{i+1}, \ldots, j_{m}\right)$ necessitates a choice of vertex $v$ from among $\left\{v_{i 1}, v_{i 2}, \ldots, v_{i j_{i}}\right\}$ in order to form $K(J)(v)$, as in Construction 1. The fundamental property, described in Definition 2.1, ensures that any choice of $v$ will result in precisely the same minimal non-simplices in $K(J)(v)=K\left(J^{\prime}\right)$.

The next theorem follows from these observations. Set $d(J)=j_{1}+j_{2}+\cdots+j_{m}$.

Theorem 2.2. Let $J=\left(j_{1}, j_{2}, \ldots, j_{m}\right)$, and suppose $K$ is dual to the boundary of a simple polytope $P^{n}$ having $m$ facets. Then $K(J)$ is dual to the boundary of a simple polytope $P(J)$ of dimension $d(J)-m+n$ having $d(J)$ facets. 
This section ends with a simple necessary criterion for a simplicial complex to be in the image of the simplicial wedge construction. The condition follows immediately from the definition of the construction.

Remark. If a simplicial complex $K^{\prime}$ exists satisfying $K=K^{\prime}(v)$ for some $\{v\} \in K^{\prime}$, then $K$ must contain vertices $v_{1}$ and $v_{2}$ satisfying:

1. the one-simplex $\left\{v_{1}, v_{2}\right\} \in K$ and

2. interchanging $v_{1}$ and $v_{2}$ is a simplicial automorphism of $K$.

\section{New toric manifolds made from a given one}

A toric manifold $M^{2 n}$ is a manifold covered by local charts $\mathbb{C}^{n}$, each with the standard action of a real $n$-dimensional torus $T^{n}$, compatible in such a way that the quotient $M^{2 n} / T^{n}$ has the structure of a simple polytope $P^{n}$. Here, "simple" means that $P^{n}$ has the property that at each vertex exactly $n$ facets intersect. Under the $T^{n}$ action, each copy of $\mathbb{C}^{n}$ must project to an $\mathbb{R}_{+}^{n}$ neighborhood of a vertex of $P^{n}$. The fundamental construction of Davis and Januszkiewicz [12, Section 1.5] is described briefly below. It realizes all toric manifolds and, in particular, all smooth projective toric varieties. Let

$$
\mathcal{F}=\left\{F_{1}, F_{2}, \ldots, F_{m}\right\}
$$

denote the set of facets of $P^{n}$. The fact that $P^{n}$ is simple implies that every codimension- $l$ face $F$ can be written uniquely as

$$
F=F_{i_{1}} \cap F_{i_{2}} \cap \cdots \cap F_{i_{l}},
$$

where the $F_{i_{j}}$ are the facets containing $F$. Let

$$
\lambda: \mathcal{F} \longrightarrow \mathbb{Z}^{n}
$$

be a function into an $n$-dimensional integer lattice satisfying the condition that whenever $F=F_{i_{1}} \cap F_{i_{2}} \cap \cdots \cap F_{i_{l}}$ then $\left\{\lambda\left(F_{i_{1}}\right), \lambda\left(F_{i_{2}}\right), \ldots, \lambda\left(F_{i_{l}}\right)\right\}$ span an $l$-dimensional submodule of $\mathbb{Z}^{n}$ that is a direct summand. Such a map is called a characteristic function associated to $P^{n}$. Next, regarding $\mathbb{R}^{n}$ as the Lie algebra of $T^{n}$, the map $\lambda$ is used to associate to each codimension- $l$ face $F$ of $P^{n}$ a rank-l subgroup $G_{F} \subset T^{n}$. Specifically, writing $\lambda\left(F_{i_{j}}\right)=\left(\lambda_{1 i_{j}}, \lambda_{2 i_{j}}, \ldots, \lambda_{n i_{j}}\right)$ gives

$$
G_{F}=\left\{\left(e^{2 \pi i\left(\lambda_{1 i_{1}} t_{1}+\lambda_{1 i_{2}} t_{2}+\cdots+\lambda_{1 i_{l}} t_{l}\right)}, \ldots, e^{2 \pi i\left(\lambda_{n i_{1}} t_{1}+\lambda_{n i_{2}} t_{2}+\cdots+\lambda_{n i_{l}} t_{l}\right)}\right) \in T^{n}\right\},
$$

where $t_{i} \in \mathbb{R}, i=1,2, \ldots, l$. Finally, let $p \in P^{n}$ and $F(p)$ be the unique face with $p$ in its relative interior. Define an equivalence relation $\sim$ on $T^{n} \times P^{n}$ by $(g, p) \sim(h, q)$ if and only if $p=q$ and $g^{-1} h \in G_{F(p)} \cong T^{l}$. Then

$$
M^{2 n} \cong M^{2 n}(\lambda)=T^{n} \times P^{n} / \sim
$$

is a smooth, closed, connected, $2 n$-dimensional manifold with $T^{n}$ action induced by left translation [12, p. 423]. A projection $\pi: M^{2 n} \rightarrow P^{n}$ onto the polytope is induced from the projection $T^{n} \times P^{n} \rightarrow P^{n}$.

Remark. In the cases when $M^{2 n}$ is a projective non-singular toric variety, $P^{n}$ and $\lambda$ encode the information in the defining fan. 
Suppose that $K$ is dual to to the boundary a simple polytope $P^{n}$ having $m$ facets. Recall that the duality here is in the sense that the facets of $P^{n}$ correspond to the vertices of $K$. A set of vertices in $K$ is a simplex if and only if the corresponding facets in $P^{n}$ all intersect. At each vertex of a simple polytope $P^{n}$, exactly $n$ facets intersect.

A characteristic function $\lambda: \mathcal{F} \longrightarrow \mathbb{Z}^{n}$ assigns an integer vector to each facet of the simple polytope $P^{n}$. It can be considered as an $(n \times m)$-matrix, $\lambda: \mathbb{Z}^{m} \longrightarrow \mathbb{Z}^{n}$, with integer entries and columns indexed by the facets of $P^{n}$. The condition following (3) may be interpreted as requiring all $n \times n$ minors of $\lambda$, corresponding to the vertices of $P^{n}$, to be \pm 1 . Given $\lambda$ and $J=\left(j_{1}, j_{2}, \ldots, j_{m}\right)$, a new function

$$
\lambda(J): \mathbb{Z}^{d(J)} \longrightarrow \mathbb{Z}^{d(J)-m+n}
$$

can be constructed by taking $\lambda(J)$ to be the $((d(J)-m+n) \times d(J))$-matrix described in Figure 1, below. In the diagram, the columns of the matrix are indexed by the vertices of $K(J)$, and $I_{k}$ denotes a $k \times k$ identity sub-matrix.

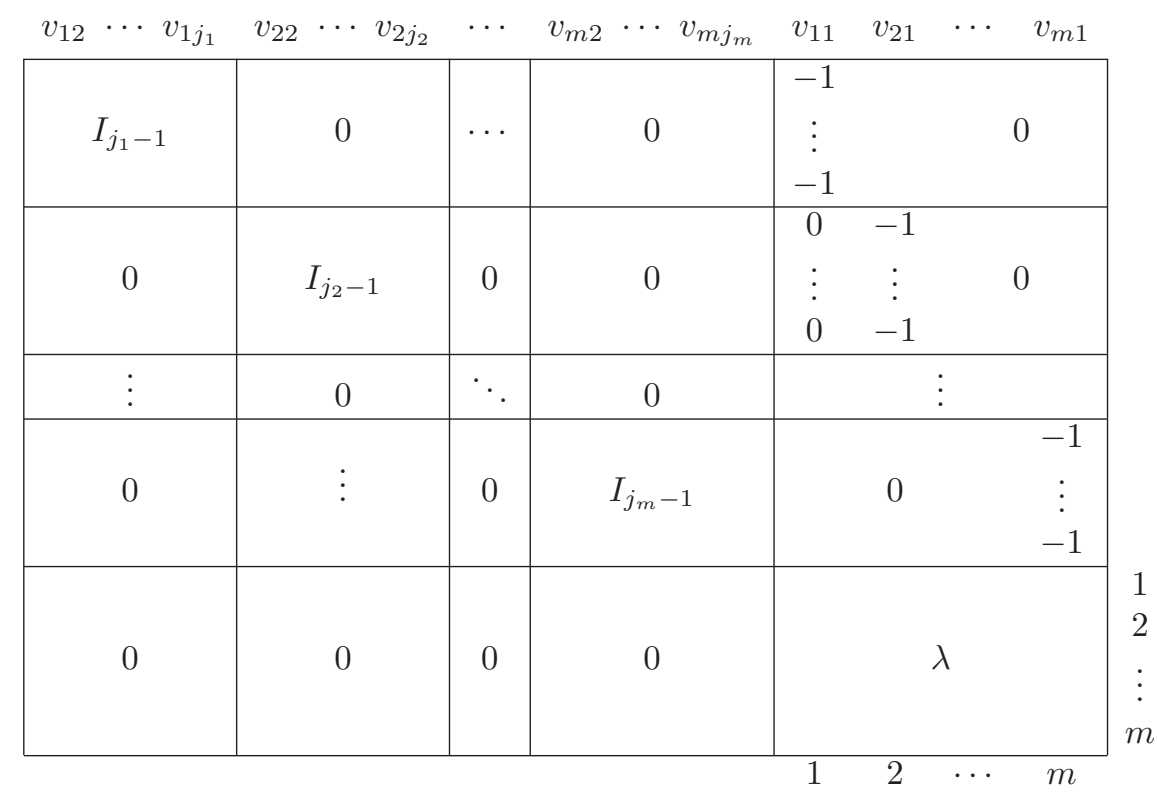

Figure 1. The matrix $\lambda(J)$

Remark 3.1. It was brought to the authors' attention by Jongbaek Song that the two $((n+2) \times(m+2))$-matrices, $\lambda(3,1, \ldots, 1)$ and $\bar{\lambda}(2,1, \ldots, 1)$, where $\bar{\lambda}=\lambda(2,1, \ldots, 1)$, differ by an element of $S L(n+2, \mathbb{Z})$. This allows for inductive arguments in this context of toric manifolds.

The next theorem constructs an infinite family of toric manifolds "derived" from the information in $\lambda, P^{n}$, and $J=\left(j_{1}, j_{2}, \ldots, j_{m}\right)$.

Theorem 3.2. If $\lambda$ is a characteristic map for a $2 n$-dimensional toric manifold $M$, then $\lambda(J)$ is the characteristic map for a toric manifold $M(J)$ of dimension $2 d(J)-$ $2 m+2 n$. 
Proof. Theorem 2.2 ensures that $P(J)$ is a simple polytope of dimension $d(J)-$ $m+n$. It remains to show that for each vertex of $P(J)$ the corresponding $(d(J)-$ $m+n) \times(d(J)-m+n)$ minor of $\lambda(J)$ is equal to \pm 1 . The proof is by induction. Consider first the case $J=(1,1, \ldots, 1, \stackrel{i}{2}, 1, \ldots, 1)$. Corresponding to the re-indexing of the vertices of $K(J)$, the facets of $P(J)$ are indexed as follows:

$$
\mathcal{F}(J)=\left\{F_{11}, F_{21}, \ldots, F_{(i-1) 1}, F_{i 1}, F_{i 2}, F_{(i+1) 1}, \ldots, F_{m 1}\right\}
$$

The matrix $\lambda(J)$ now has the form

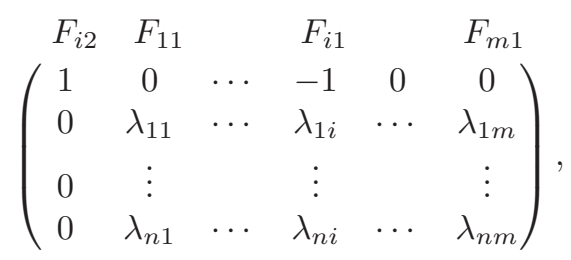

where $\lambda=\left(\lambda_{i j}\right)$ is the original matrix. (Recall that vertices $v_{i k}$ of $K(J)$ correspond to facets $F_{i k}$ of the polytope $P(J)$.) The minors corresponding to the new $(n+1)$-fold intersections of facets, are of two types:

1. those which include columns indexed by both $F_{i 1}$ and $F_{i 2}$, and

2. those which include columns indexed by either $F_{i 1}$ or $F_{i 2}$ but not both.

This observation follows from the fact that the simplicial wedge construction ensures that each new maximal simplex of $K(J)=K\left(v_{i}\right)$ must contain either $v_{i 1}$ or $v_{i 2}$. According to the discussion following Construction 1, the first type arise from intersections

$$
F_{i_{1}} \cap F_{i_{2}} \cap \cdots \cap F_{i_{k}} \cap F_{i} \cap F_{i_{k+1}} \cap \cdots \cap F_{i_{n}}
$$

of $n$ facets in $P^{n}$. In $P(J)$, they give $(n+1)$-fold intersections

$$
F_{i_{1} 1} \cap F_{i_{2} 1} \cap \cdots \cap F_{i_{k} 1} \cap F_{i 1} \cap F_{i 2} \cap F_{i_{(k+1) 1}} \cap \cdots \cap F_{i_{n} 1} .
$$

The corresponding $(n+1) \times(n+1)$ minors in the matrix $\lambda(J)$ above are

$$
\left|\begin{array}{cccccccc}
F_{i 2} & F_{i_{1} 1} & & F_{i_{k} 1} & F_{i 1} & F_{i_{k+1} 1} & & F_{i_{n} 1} \\
1 & 0 & \cdots & 0 & -1 & 0 & 0 & 0 \\
0 & \lambda_{1 i_{1}} & \cdots & \lambda_{1 i_{k}} & \lambda_{1 i} & \lambda_{1 i_{k+1}} & \cdots & \lambda_{1 i_{n}} \\
\vdots & \vdots & & \vdots & \vdots & \vdots & & \vdots \\
0 & \lambda_{n i_{1}} & \cdots & \lambda_{n i_{k}} & \lambda_{n i} & \lambda_{1 i_{k+1}} & \cdots & \lambda_{n i_{n}}
\end{array}\right| .
$$

Expanding by the first row gives \pm 1 by $(5)$. Now $(n+1)$-fold intersections of facets of the second type, which contain $F_{i 2}$ but not $F_{i 1}$ (or vice versa) arise from intersections in $\mathrm{P}$ that do not involve the facet $F_{i}$. That is, they are of the form

$$
F_{i_{1}} \cap F_{i_{2}} \cap \cdots \cap F_{i_{k}} \cap F_{i_{k+1}} \cap \cdots \cap F_{i_{n}}, \quad i_{j} \neq i .
$$

In $P(J)$, the intersection is

$$
F_{12} \cap F_{i_{1} 1} \cap F_{i_{2} 1} \cap \cdots \cap F_{i_{k} 1} \cap F_{i_{(k+1) 1}} \cap \cdots \cap F_{i_{n} 1}, \quad i_{j} \neq i .
$$


The $(n+1) \times(n+1)$ minor in $\lambda(J)$ will have the form

$$
\begin{array}{cccc}
F_{i 2} & F_{i_{1} 1} & & F_{i_{n} 1} \\
1 & 0 & \cdots & 0 \\
0 & \lambda_{1 i_{1}} & \cdots & \lambda_{1 i_{n}} \\
\vdots & \vdots & & \vdots \\
0 & \lambda_{n i_{1}} & \cdots & \lambda_{n i_{n}}
\end{array} \mid
$$

and so will have the value \pm 1 . Finally, $(n+1)$-fold intersections of facets of the second type, which contain $F_{i 1}$ but not $F_{i 2}$ again arise from intersections of the type 6 . In $P(J)$ the intersection is

$$
F_{i_{1} 1} \cap F_{i_{2} 1} \cap \cdots \cap F_{i_{k} 1} \cap F_{i 1} \cap F_{i_{(k+1) 1}} \cap \cdots \cap F_{i_{n} 1} .
$$

and the corresponding $(n+1) \times(n+1)$ minor in $\lambda(J)$ has the form

$$
\left|\begin{array}{ccccccc}
F_{i_{1} 1} & & F_{i_{k} 1} & F_{i 1} & F_{i_{k+1} 1} & & F_{i_{n} 1} \\
0 & \cdots & 0 & -1 & 0 & 0 & 0 \\
\lambda_{1 i_{1}} & \cdots & \lambda_{1 i_{k}} & \lambda_{1 i} & \lambda_{1 i_{k+1}} & \cdots & \lambda_{1 i_{n}} \\
\vdots & & \vdots & \vdots & \vdots & & \vdots \\
\lambda_{n i_{1}} & \cdots & \lambda_{n i_{k}} & \lambda_{n i} & \lambda_{1 i_{k+1}} & \cdots & \lambda_{n i_{n}}
\end{array}\right| .
$$

Expansion by the first row gives the $n \times n$ minor in $\lambda$

$$
\left|\begin{array}{cccccc}
\lambda_{1 i_{1}} & \cdots & \lambda_{1 i_{k}} & \lambda_{1 i_{k+1}} & \cdots & \lambda_{1 i_{n}} \\
\vdots & & \vdots & \vdots & & \vdots \\
\lambda_{n i_{1}} & \cdots & \lambda_{n i_{k}} & \lambda_{1 i_{k+1}} & \cdots & \lambda_{n i_{n}}
\end{array}\right|,
$$

which has the value \pm 1 because it corresponds to (6). The inductive step passes from the $m$-tuple $J=\left(j_{1}, j_{2}, \ldots, j_{m}\right)$ to $J^{\prime}=\left(j_{1}, j_{2}, \ldots, j_{k-1}, j_{k}+1, j_{k+1} \ldots, j_{m}\right)$ and follows the same argument, replacing the characteristic map $\lambda$ in the discussion above with $\lambda(J)$, This completes the proof.

Remark 3.3. In a recent preprint [5], the authors show that the manifolds $M(J)$ can be obtained alternatively by a reinterpretation and generalization of the DavisJanuszkiewicz construction (4).

\section{The cohomology of the toric manifolds $M(J)$}

The rows of the matrix $\lambda(J)$ determine an ideal $L_{M(J)}$ generated by linear relations among the generators of the Stanley-Reisner ring of $K(J)$. These are given by

$$
\begin{aligned}
v_{i t}-v_{i 1} & =0, \quad t=2, \ldots, j_{i}, \quad i=1, \ldots, m \\
\lambda_{i 1} v_{11}+\lambda_{i 2} v_{21}+\cdots+\lambda_{i m} v_{m 1} & =0, \quad i=1, \ldots, n .
\end{aligned}
$$

Notice that the second set of relations are those corresponding to the linear ideal determined by the matrix $\lambda$. The next result is the Davis-Januszkiewicz (DanilovJurkewicz) theorem [12, Theorem 4.14] for the toric manifold $M(J)$. 
Theorem 4.1. The cohomology ring $H^{*}(M(J) ; \mathbb{Z})$ is isomorphic to

$$
\mathbb{Z}\left[v_{11}, v_{12}, \ldots, v_{1 j_{1}}, v_{21}, v_{22}, \ldots, v_{2 j_{2}}, \ldots, v_{m 1}, v_{m 2}, \ldots, v_{m j_{m}}\right] /\left(I_{K(J)}+L_{M(J)}\right),
$$

where $I_{K(J)}$ denotes the Stanley-Reisner ideal for the simplicial complex $K(J)$.

Applying the linear relations (7) and rewriting $v_{i 1}$ as $v_{i}$ allows a significant simplification of this description.

Theorem 4.2. The cohomology ring $H^{*}(M(J) ; \mathbb{Z})$ is isomorphic to

$$
\mathbb{Z}\left[v_{1}, v_{2}, \ldots, v_{m}\right] /\left(I_{K}^{J}+L_{M}\right),
$$

where each $v_{i}$ has degree 2, $L_{M}$ is the ideal in the Stanley-Reisner ring of $K$ generated by the rows of the matrix $\lambda$ and $I_{K}^{J}$ is the ideal of relations generated by all monomials of the form

$$
v_{i_{1}}^{j_{i_{1}}} v_{i_{2}}^{j_{i_{2}}} \cdots v_{i_{k}}^{j_{i_{k}}}
$$

corresponding to the minimal non-simplex $\left\{v_{i_{1}}, v_{i_{2}}, \ldots, v_{i_{k}}\right\}$ of $K$.

Proof. Linear relations (7) and the relabeling of $v_{i 1}$ as $v_{i}$ convert the monomials generating $I_{K(J)}$ into those of (8) and the relations $L_{M(J)}$ into the relations $L_{M}$.

\section{A class of examples}

In this section is constructed a family of toric manifolds beginning with the example $M^{4}$ for which the simple polytope $P^{2}$ is the two-dimensional square having four facets and the characteristic map $\lambda$ is given by the $2 \times 4$-matrix

$$
\lambda=\left(\begin{array}{llll}
1 & -1 & 1 & 0 \\
2 & -1 & 0 & 1
\end{array}\right)
$$

The cohomology of $M^{4}$ is computed from the Davis-Januszkiewicz theorem [12, Theorem 4.14]. It is the quotient of the polynomial ring $\mathbb{Z}\left[v_{1}, v_{2}, v_{3}, v_{4}\right]$ on four twodimensional generators by a linear ideal $L_{M^{4}}$ and a monomial ideal $I_{K}$, where $K$ is the simplicial complex dual to $\partial P^{2}$. The ideal $L_{M^{4}}$ is generated by $v_{1}-v_{2}+v_{3}$ and $2 v_{1}-v_{2}+v_{4}$, and the ideal $I_{K}$ is generated by $v_{1} v_{3}$ and $v_{2} v_{4}$. These relations give the computation

$$
H^{*}\left(M^{4}\right) \cong \mathbb{Z}\left[v_{1}, v_{3}\right] /\left\langle v_{1} v_{3}, v_{1}^{2}=v_{3}^{2}\right\rangle .
$$

This is the cohomology ring of the connected sum $\mathbb{C} P^{2} \# \mathbb{C} P^{2}$, so the cohomological rigidity results of $[\mathbf{1 1}]$ imply that $M^{4}$ is homeomorphic to $\mathbb{C} P^{2} \# \mathbb{C} P^{2}$.

Next, take $J=(1, p, 1, q)$ to get $M(J)\left(=M^{4}(J)\right)$. Theorem 4.2 gives $H^{*}(M(J))$ as the quotient of the polynomial ring $\mathbb{Z}\left[v_{1}, v_{2}, v_{3}, v_{4}\right]$ by the linear ideal $L_{M^{4}}$ and a monomial ideal $I_{K}^{J}$. As before, the ideal $L_{M^{4}}$ is generated by $v_{1}-v_{2}+v_{3}$ and $2 v_{1}-v_{2}+v_{4}$, and the ideal $I_{K}^{J}$ is generated by $v_{1} v_{3}$ and $v_{2}^{p} v_{4}^{q}$. These relations are 
simplified by using the fact that $v_{1} v_{3}=0$ to get

$$
\left(v_{3}-v_{1}\right)^{p}=v_{3}^{p}+(-1)^{p} v_{1}^{p} \text { and }\left(v_{3}+v_{1}\right)^{q}=v_{3}^{q}+v_{1}^{q} .
$$

Writing $0=v_{2}^{p} v_{4}^{q}=\left(v_{3}-v_{1}\right)^{p}\left(v_{3}+v_{1}\right)^{q}$ gives

$$
0=\left(v_{3}^{p}+(-1)^{p} v_{1}^{p}\right)\left(v_{3}^{q}+v_{1}^{q}\right)=v_{3}^{p+q}+(-1)^{p} v_{1}^{p+q} .
$$

Thus $v_{3}^{p+q}=(-1)^{p+1} v_{1}^{p+q}$, giving

$$
H^{*}(M(J)) \cong \mathbb{Z}\left[v_{1}, v_{3}\right] /\left\langle v_{1} v_{3}, v_{3}^{p+q}=(-1)^{p+1} v_{1}^{p+q}\right\rangle .
$$

This is the cohomology ring of the connected sum $\mathbb{C} P^{p+q} \#(-1)^{p} \mathbb{C} P^{p+q}$, where, here, the $(-1)^{p}$ indicates an orientation change with $p$. Again the cohomological rigidity results of $[\mathbf{1 1}]$ imply that $M^{J}$ is homeomorphic to $\mathbb{C} P^{p+q} \#(-1)^{p} \mathbb{C} P^{p+q}$.

Remark. The situation becomes more complex for general $J=\left(j_{1}, j_{2}, j_{3}, j_{4}\right)$, with all $j_{i}>1$. The "heights" of $v_{1}$ and $v_{3}$ are harder to determine. It would interesting to know if for this particular manifold $M^{4}$, non-trivial examples of sequences $J$ and $J^{\prime}$ exist so that $M(J)$ and $M\left(J^{\prime}\right)$ have isomorphic cohomology rings.

J. Song and S. Choi have produced examples of non-diffeomorphic toric manifolds $M$ and $N$ and a sequence $J$ so that $M(J)$ and $N(J)$ are diffeomorphic.

\section{Polyhedral products}

Let $K$ be a simplicial complex with $m$ vertices, and let $(\underline{X}, \underline{A})$ denote a family of CW pairs

$$
\left(X_{1}, A_{1}\right),\left(X_{2}, A_{2}\right), \ldots,\left(X_{m}, A_{m}\right) .
$$

When all the pairs $\left(X_{i}, A_{i}\right)$ are the same pair $(X, A)$, the family $(\underline{X}, \underline{A})$ is written simply as $(X, A)$. A polyhedral product is a topological space

$$
Z(K ;(\underline{X}, \underline{A})) \subseteq \prod_{i=1}^{m} X_{i},
$$

defined as a colimit by a diagram $D: K \rightarrow C W_{*}$. At each $\sigma \in K$, it is given by

$$
D(\sigma)=\prod_{i=1}^{m} W_{i}, \quad \text { where } \quad W_{i}=\left\{\begin{array}{lll}
X_{i} & \text { if } & i \in \sigma \\
A_{i} & \text { if } & i \in[m]-\sigma .
\end{array}\right.
$$

Here, the colimit is a union given by

$$
Z(K ;(\underline{X}, \underline{A}))=\bigcup_{\sigma \in K} D(\sigma) .
$$

Detailed background information about polyhedral products may be found in $[\mathbf{7}, \mathbf{9}$, 1, 2] (and in the unpublished notes of N. Strickland).

The family of $C W$-pairs $(\underline{X}, \underline{A})$ to be investigated here is specified by the sequence of positive integers $J=\left(j_{1}, \ldots, j_{m}\right)$ and is given by

$$
(\underline{X}, \underline{A})=\left(\underline{D}^{2 J}, \underline{S}^{2 J-1}\right)=\left\{\left(D^{2 j_{i}}, S^{2 j_{i}-1}\right)\right\}_{i=1}^{m} .
$$

(Presently, it will become necessary to consider the discs $D^{2 j_{i}}$ as embedded naturally in $\mathbb{C}^{j_{i}}$.) If $J=(1,1, \ldots, 1)$, the space $Z\left(K ;\left(\underline{D}^{2 J}, \underline{S}^{2 J-1}\right)\right)$ is an ordinary momentangle complex and is written $Z\left(K ;\left(D^{2}, S^{1}\right)\right)$. 
For fixed $K$, the spaces $Z\left(K ;\left(\underline{D}^{2 J}, \underline{S}^{2 J-1}\right)\right)$ all have the property of being stably wedge equivalent.

Definition 6.1. Two spaces $X$ and $Y$ are said to be stably wedge equivalent if there are stable homotopy equivalences

$$
X \sim X_{1} \vee X_{2} \vee \ldots \vee X_{t} \text { and } Y \sim Y_{1} \vee Y_{2} \vee \ldots \vee Y_{t}
$$

and $X_{i}$ is stably homotopy equivalent to $Y_{i}$ for all $i=1,2, \ldots, t$.

The next proposition follows directly from the stable splitting theorems for generalized moment-angle complexes of [1], [2, Corollary 2.24] and [3, Theorem 1.4] that describes the cohomology ring structure in terms of the stable splitting.

Proposition 6.2. For fixed $K$ and all $J=\left(j_{1}, \ldots, j_{m}\right)$, the spaces $Z\left(K ;\left(\underline{D}^{2 J}, \underline{S}^{2 J-1}\right)\right)$ are all stably wedge equivalent, and, moreover, they have isomorphic ungraded cohomology rings.

\section{The simplicial wedge construction and polyhedral products}

Recall that a product of CW pairs is defined by

$$
(X, A) \times(Y, B):=(X \times Y,(X \times B) \cup(A \times Y)) .
$$

The $k$-fold iteration $(X, A) \times \cdots \times(X, A)$ is denoted by $(X, A)^{k}$.

Let $K$ be a simplicial complex on $m$ vertices $\left\{v_{1}, v_{2}, \ldots, v_{m}\right\}$, and let $(\underline{X}, \underline{A})$ denote the family of CW pairs

$$
\left(X_{1}, A_{1}\right),\left(X_{2}, A_{2}\right), \ldots,\left(X_{m}, A_{m}\right) .
$$

In light of Definition 2.1, it becomes necessary at this point to introduce a notational convention to avoid expressions becoming too unwieldy.

Convention. Let $J=\left(j_{1}, j_{2}, \ldots, j_{m}\right)$ be sequence of positive integers $K(J)$ as in Definition 2.1 and the family of pairs $(\underline{X}, \underline{A})$ as above. Denote by $Z(K(J) ;(\underline{X}, \underline{A}))$ the polyhedral product determined by the simplicial complex $K(J)$ and the family of pairs obtained from $(\underline{X}, \underline{A})$ by repeating each $\left(X_{i}, A_{i}\right), j_{i}$ times in sequence.

Fix $i \in\{1,2, \ldots, m\}$, and define a family of CW pairs $(\underline{Y}, \underline{B})$ by

$$
\left(Y_{k}, B_{k}\right)=\left\{\begin{array}{lll}
\left(X_{k}, A_{k}\right) & \text { if } & k \neq i \\
\left(X_{i}, A_{i}\right)^{2} & \text { if } \quad k=i .
\end{array}\right.
$$

Theorem 7.1. The polyhedral product spaces $Z(K ;(\underline{Y}, \underline{B}))$ and $Z\left(K\left(v_{i}\right) ;(\underline{X}, \underline{A})\right)$ are equal subspaces of $W_{1} \times \cdots \times W_{m}$, where

$$
W_{k}=\left\{\begin{array}{lll}
X_{k} & \text { if } & k \neq i \\
X_{k} \times X_{k}, & \text { if } & k=i
\end{array}\right.
$$

Proof. As in Construction 1, the vertices of $K\left(v_{i}\right)$ are

$$
S=\left\{v_{11}, \ldots, v_{(i-1) 1}, v_{i 1}, v_{i 2}, v_{(i+1) 1}, \ldots, v_{m 1}\right\} .
$$

Let $\sigma=\left\{v_{t_{1}}, v_{t_{2}}, \ldots, v_{t_{k}}\right\}$ be a maximal simplex in $K$. If $v_{i}=v_{t_{s}} \in \sigma$, then $D(\sigma)$ is equal to $D\left(\sigma^{\prime}\right) \subset Z\left(K\left(v_{i}\right) ;(\underline{X}, \underline{A})\right)$, where 


$$
\sigma^{\prime}=\left\{v_{t_{1} 1}, v_{t_{2} 1}, \ldots, v_{\left(t_{s}-1\right) 1}, v_{t_{s} 1}, v_{t_{s} 2}, v_{\left(t_{s}+1\right) 1}, \ldots, v_{t_{k} 1}\right\} .
$$

If $v_{i} \notin \sigma$, then $D(\sigma)$ is identified (by the identity map) with $D\left(\sigma_{1}^{\prime}\right) \cup D\left(\sigma_{2}^{\prime}\right) \subset$ $Z\left(K\left(v_{i}\right) ;(\underline{X}, \underline{A})\right)$, where

$$
\begin{aligned}
& \sigma_{1}^{\prime}=\left\{v_{t_{1} 1}, v_{t_{2} 1}, \ldots, v_{t_{k} 1}, v_{i 1}\right\}, \\
& \sigma_{2}^{\prime}=\left\{v_{t_{1} 1}, v_{t_{2} 1}, \ldots, v_{t_{k} 1}, v_{i 2}\right\} .
\end{aligned}
$$

(Here, the vertices may not be in their correct order.) The fact that the wedge construction ensures that the maximal simplices $\sigma_{1}^{\prime}$ and $\sigma_{2}^{\prime}$ exist in $K\left(v_{i}\right)$ has been used here. This procedure exhausts all maximal simplices in $K\left(v_{i}\right)$ and completes the equivalence.

The next iteration of this procedure is slightly more involved but serves to describe all iterations. Choose $j \in\{1,2, \ldots, m\}$. If $j \neq i$, the new family $(\underline{Y}, \underline{B})$ is defined by

$$
\left(Y_{k}, B_{k}\right)=\left\{\begin{array}{lll}
\left(X_{k}, A_{k}\right) & \text { if } & k \neq i, j \\
\left(X_{k}, A_{k}\right)^{2} & \text { if } & k=i, j .
\end{array}\right.
$$

In this case, $K\left(v_{i}\right)$ is replaced with $\left(K\left(v_{i}\right)\right)\left(v_{j 1}\right)$ and the procedure is exactly as described above. The result is that $Z(K ;(\underline{Y}, \underline{B}))$ and $Z\left(\left(K\left(v_{i}\right)\right)\left(v_{j 1}\right) ;(\underline{X}, \underline{A})\right)$ are equivalent subspaces of $W_{1} \times \cdots \times W_{m}$, where

$$
W_{k}=\left\{\begin{array}{lll}
X_{k} & \text { if } \quad k \neq i, j \\
X_{k} \times X_{k}, & \text { if } \quad k=i, j
\end{array}\right.
$$

In the case $j=i$, the new family $(\underline{Y}, \underline{B})$ will have

$$
\left(Y_{k}, B_{k}\right)=\left\{\begin{array}{lll}
\left(X_{k}, A_{k}\right) & \text { if } & k \neq i \\
\left(X_{i}, A_{i}\right)^{3} & \text { if } & k=i .
\end{array}\right.
$$

In this case, $K\left(v_{i}\right)$ is replaced with either $\left(K\left(v_{i}\right)\right)\left(v_{i 1}\right)$ or $\left(K\left(v_{i}\right)\right)\left(v_{i 2}\right)$. The symmetry of the wedge construction ensures that these two simplicial complexes are isomorphic as simplicial complexes. The result is that $Z(K ;(\underline{Y}, \underline{B}))$ and $Z\left(\left(K\left(v_{i}\right)\right)\left(v_{i 1}\right) ;(\underline{X}, \underline{A})\right)$ are equivalent subspaces of $W_{1} \times \cdots \times W_{m}$, where

$$
W_{k}=\left\{\begin{array}{lll}
X_{k} & \text { if } & k \neq i \\
\prod_{s=1}^{3} X_{k} & \text { if } & k=i
\end{array}\right.
$$

The general iteration procedure is now straightforward; the result is recorded in the next theorem.

Theorem 7.2. Let $K$ be a simplicial complex with $m$ vertices, and let $(\underline{X}, \underline{A})$ denote a family of $C W$ pairs

$$
\left\{\left(X_{1}, A_{1}\right),\left(X_{2}, A_{2}\right), \ldots,\left(X_{m}, A_{m}\right)\right\} .
$$

For $J=\left(j_{1}, j_{2}, \ldots, j_{m}\right)$, a sequence of positive integers, define a new family of pairs

$$
\bar{J}(\underline{X}, \underline{A})=(\underline{Y}, \underline{B})) \quad \text { by } \quad\left(Y_{k}, B_{k}\right)=\left(X_{k}, A_{k}\right)^{j_{k}}, \quad k=1,2, \ldots, m .
$$

Then, the polyhedral product spaces $Z(K ; \bar{J}(\underline{X}, \underline{A}))$ and $Z(K(J) ;(\underline{X}, \underline{A}))$ are equal subspaces of $X_{1}^{j_{1}} \times X_{2}^{j_{2}} \times \cdots \times X_{m}^{j_{m}}$. 
This result is applied to the family where $\left(X_{i}, A_{i}\right)=\left(D^{2}, S^{1}\right)$ for all $i=1,2, \ldots, m$. In this case,

$$
\left(Y_{k}, B_{k}\right)=\left(X_{k}, A_{k}\right)^{j_{k}}=\left(\left(D^{2}\right)^{j_{k}}, \partial\left(\left(D^{2}\right)^{j_{k}}\right)\right),
$$

where $\partial\left(\left(D^{2}\right)^{j_{i}}\right)$ denotes the boundary of a $j_{i}$-fold product of two-discs. For this particular case, the family $(\underline{Y}, \underline{B})$ is denoted by

$$
\left(\underline{B}^{2 J}, \underline{\partial B}^{2 J}\right):=\left\{\left(\left(D^{2}\right)^{j_{i}}, \partial\left(\left(D^{2}\right)^{j_{i}}\right)\right\}_{i=1}^{m} .\right.
$$

Theorem 7.2 implies now the next corollary.

Corollary 7.3. The generalized moment-angle complex $Z\left(K ;\left(\underline{B}^{2 J}, \underline{\partial B}^{2 J}\right)\right)$ and the moment-angle complex $Z\left(K(J) ;\left(D^{2}, S^{1}\right)\right)$ are equal subspaces of

$$
\left(D^{2}\right)^{j_{1}} \times\left(D^{2}\right)^{j_{2}} \times \cdots \times\left(D^{2}\right)^{j_{m}}=\left(D^{2}\right)^{d(J)} .
$$

Remark. Notice that by considering $\left(D^{2}\right)^{d(J)} \subset \mathbb{C}^{d(J)}$, both moment-angle complexes inherit an action of the real torus $T^{d(J)}$ with respect to which they are equivariantly equivalent.

An entirely similar argument shows that taking $\left(X_{i}, A_{i}\right)=\left(D^{1}, S^{0}\right)$ for all $i=$ $1, \ldots, m$ and $J=(2,2, \ldots, 2)$, yields the result that the spaces $Z\left(K ;\left(D^{1} \times D^{1}\right.\right.$, $\left.\partial\left(D^{1} \times D^{1}\right)\right)$ and $Z\left(K(J) ;\left(D^{1}, S^{0}\right)\right)$ are equivalent subspaces of $\left(D^{1}\right)^{2} \times\left(D^{1}\right)^{2} \times$ $\cdots \times\left(D^{1}\right)^{2}$. It follows by the arguments below that $Z\left(K ;\left(D^{2}, S^{1}\right)\right)$ and $Z(K(J)$; $\left.\left(D^{1}, S^{0}\right)\right)$ are diffeomorphic.

Recall now the family of pairs $\left(\underline{D}^{2 J}, \underline{S}^{2 J-1}\right)$ described in (10). Observe that for the corresponding generalized moment-angle complex there is a natural embedding

$$
Z\left(K ;\left(\underline{D}^{2 J}, \underline{S}^{2 J-1}\right)\right) \subseteq D^{2 j_{1}} \times D^{2 j_{2}} \times \cdots \times D^{2 j_{m}}
$$

The next goal is to verify that the spaces $Z\left(K ;\left(\underline{B}^{2 J}, \underline{\partial B}^{2 J}\right)\right)$ and $Z\left(K ;\left(\underline{D}^{2 J}, \underline{S}^{2 J-1}\right)\right)$ are equivariantly homeomorphic with respect to various torus actions.

Let $\left(D^{2}\right)^{j_{i}} \subset \mathbb{C}^{j_{i}}$ be embedded in the usual way, and choose a standard homeomorphisms of pairs $h_{i}:\left(\left(D^{2}\right)^{j_{i}}, \partial\left(\left(D^{2}\right)^{j_{i}}\right) \longrightarrow\left(D^{2 j_{i}}, S^{2 j_{i}-1}\right)\right.$. Define an action of the circle $T^{1}$ on $D^{2 j_{i}}$ by

$$
t \cdot h_{i}\left(z_{1}, z_{2}, \ldots, z_{j_{i}}\right)=h_{i}\left(t z_{1}, t z_{2}, \ldots, t z_{j_{i}}\right) .
$$

Next, denote the $j_{i}$-tuple $\left(z_{1}, z_{2}, \ldots, z_{j_{i}}\right)$ by the symbol $\underline{z}_{j_{i}}$ and define an action of $T^{m}$ on $\left(D^{2}\right)^{d(J)}$ by

$$
\left(t_{1}, t_{2}, \ldots, t_{m}\right) \cdot\left(\underline{z}_{j_{1}}, \underline{z}_{j_{2}}, \ldots, \underline{z}_{j_{m}}\right)=\left(t_{1} \underline{z}_{j_{1}}, t_{2} \underline{z}_{j_{2}}, \ldots, t_{m} \underline{z}_{j_{m}}\right)
$$

where $t_{i} \underline{z}_{j_{i}}$ has the usual meaning $\left(t_{i} z_{1}, t_{i} z_{2}, \ldots, t_{i} z_{j_{i}}\right)$. Notice that this action of $T^{m}$ is a restriction of the natural action of the torus $T^{d(J)}$ on $\left(D^{2}\right)^{d(J)}$. An action of $T^{m}$ is induced on $D^{2 j_{1}} \times D^{2 j_{2}} \times \cdots \times D^{2 j_{m}}$ by

$$
\begin{aligned}
\left(t_{1}, t_{2}, \ldots, t_{m}\right) \cdot\left(h_{1}\left(\underline{z}_{j_{1}}\right), h_{2}\left(\underline{z}_{j_{2}}\right), \ldots,\right. & \left.h_{m}\left(\underline{z}_{j_{m}}\right)\right) \\
& =\left(h_{1}\left(t_{1} \underline{z}_{j_{1}}\right), h_{2}\left(t_{2} \underline{z}_{j_{2}}\right), \ldots, h_{m}\left(t_{m} \underline{z}_{j_{m}}\right)\right) .
\end{aligned}
$$

The homeomorphisms $h_{i}$ give rise to a homeomorphism, equivariant with respect to 
the $T^{m}$-action above,

$$
H:\left(D^{2}\right)^{d(J)} \longrightarrow D^{2 j_{1}} \times D^{2 j_{2}} \times \cdots \times D^{2 j_{m}}
$$

by $H\left(\underline{z}_{j_{1}}, \underline{z}_{j_{2}}, \ldots, \underline{z}_{j_{m}}\right)=\left(h_{1}\left(\underline{z}_{j_{1}}\right), h_{2}\left(\underline{z}_{j_{2}}\right), \ldots, h_{m}\left(\underline{z}_{j_{m}}\right)\right)$. The map $H$ extends to a homeomorphism of generalized moment-angle complexes.

Lemma 7.4. The homeomorphism $H$ extends to a homeomorphism of generalized moment-angle complexes $Z\left(K ;\left(\underline{B}^{2 J}, \underline{\partial B}^{2 J}\right)\right)$ and $Z\left(K ;\left(\underline{D}^{2 J}, \underline{S}^{2 J-1}\right)\right)$, which is equivariant with respect to the $T^{m}$-action defined by (13).

Proof. The map $H$ induces a homeomorphisms at the level of the appropriate spaces $D(\sigma)$ defined in $(9)$, compatible with the maps defining both colimits.

Combining this observation with Corollary 7.3 and the remark following it yields a key result.

Theorem 7.5. There is an action of $T^{m}$ on polyhedral products $Z\left(K ;\left(\underline{D}^{2 J}, \underline{S}^{2 J-1}\right)\right)$ and $Z\left(K(J) ;\left(D^{2}, S^{1}\right)\right)$ with respect to which they are equivariantly homeomorphic.

When combined with Theorem 1.8 of [3], this theorem yields an immediate corollary.

Corollary 7.6. The spaces $Z\left(K ;\left(D^{2}, S^{1}\right)\right)$ and $Z\left(K(J) ;\left(D^{2}, S^{1}\right)\right)$ have isomorphic ungraded cohomology rings.

These results yield an observation about the action of the Steenrod algebra.

Corollary 7.7. There is an isomorphism of ungraded $\mathbb{Z} / 2$-modules

$$
H^{*}\left(Z\left(K ;\left(D^{2}, S^{1}\right)\right) ; \mathbb{Z} / 2\right) \longrightarrow H^{*}\left(Z\left(K(J) ;\left(D^{2}, S^{1}\right)\right) ; \mathbb{Z} / 2\right)
$$

that commutes with the action of the Steenrod algebra.

Proof. The Steenrod operations are stable operations, and hence the splitting theorem [2, Theorem 2.21] implies that there is an isomorphism of ungraded $\mathbb{Z} / 2$-modules

$$
H^{*}\left(Z\left(K ;\left(D^{2}, S^{1}\right)\right) ; \mathbb{Z} / 2\right) \longrightarrow H^{*}\left(Z\left(K ;\left(\underline{D}^{2 J}, \underline{S}^{2 J-1}\right)\right) ; \mathbb{Z} / 2\right)
$$

that commutes with the action of the Steenrod algebra. The result follows from Theorem 7.5 .

Remark. Corollary 7.7 holds equally well for $\mathbb{Z} / p$ with $p$ an odd prime.

\section{A generalization to topological joins}

As usual, let $K$ be a simplicial complex on $m$ vertices, and let $J=\left(j_{1}, j_{2}, \ldots, j_{m}\right)$, be a sequence of positive integers. Consider the family of pairs

$$
(\underline{C X}, \underline{X})=\left\{\left(C X_{i}, X_{i}\right)\right\}_{i=1}^{m},
$$

where each space $X_{i}$ is a CW complex and $C X_{i}$ denotes the cone on $X_{i}$. Applying Theorem 7.2 to this family of pairs yields an equivalence of polyhedral products

$$
Z(K ; \bar{J}(\underline{C X}, \underline{X})) \longrightarrow Z(K(J) ;(\underline{C X}, \underline{X}))
$$

where, as before, $\bar{J}(\underline{C X}, \underline{X})=(\underline{Y}, \underline{B})$ with $\left(Y_{k}, B_{k}\right)=\left(C X_{k}, X_{k}\right)^{j_{k}}, k=1,2, \ldots, m$. The equivalence is as subspaces of $\left(C X_{1}\right)^{j_{1}} \times\left(C X_{2}\right)^{j_{2}} \times \cdots \times\left(C X_{m}\right)^{j_{m}}$. The next lemma, which is well known, is a key ingredient. 
Lemma 8.1. For any finite $C W$ complex $X$, there is a homeomorphism of pairs

$$
(C(X * X), X * X) \stackrel{f}{\longrightarrow}(C X, X)^{2},
$$

where $*$ denotes the topological join.

Proof. Represent a point in $C(X * X)$ by $\left[s,\left[x_{1}, t, x_{2}\right]\right]$. Define the homeomorphism $f$ by

$$
f\left(\left[s,\left[x_{1}, t, x_{2}\right]\right]\right)=\left(\left[2 s \cdot \min \{t, 1 / 2\}, x_{1}\right],\left[2 s \cdot \min \{1-t, 1 / 2\}, x_{2}\right]\right) \in C X \times C X,
$$

where the cone point is at $s=0$. At $s=1, f$ is the usual homeomorphism

$$
X * X \longrightarrow(C X \times X) \cup(X \times C X) .
$$

The map $f$ is a continuous bijection between compact Hausdorff spaces and hence is a homeomorphism.

Next, define a family of CW pairs by

$$
\left(\underline{C\left({\aleph_{J} X}_{J}\right)}, \underline{\aleph_{J} X}\right):=\left\{(C(\underbrace{X_{i} * X_{i} * \cdots * X_{i}}_{j_{i}}), \underbrace{X_{i} * X_{i} * \cdots * X_{i}}_{j_{i}})\right\}_{i=1}^{m} .
$$

The map $f$ of Lemma 8.1 iterates easily to produce a homeomorphism of pairs

$$
(C(\underbrace{X_{k} * X_{k} * \cdots * X_{k}}_{j_{k}}), \underbrace{X_{k} * X_{k} * \cdots * X_{k}}_{j_{k}}) \stackrel{f_{j_{k}}}{\longrightarrow}\left(C X_{k}, X_{k}\right)^{j_{k}},
$$

which extends to a map of families of pairs

$$
\left.\left(\underline{C\left(*_{J} X\right)}, \underline{*_{J} X}\right) \stackrel{f_{J}}{\longrightarrow}(\underline{Y}, \underline{B})\right) .
$$

The results above combine now to give the next theorem.

Theorem 8.2. There is a homeomorphism of polyhedral products

$$
\left.Z\left(K ;\left(\underline{C\left(*_{J} X\right.}\right), \underline{*_{J} X}\right)\right) \longrightarrow Z(K(J) ;(\underline{C X}, \underline{X})) .
$$

Proof. The homeomorphism is given by composing the homeomorphism of polyhedral products induced by $f_{J}$ with the homeomorphism of (15).

\section{Toric manifolds and generalized moment-angle complexes}

The information in a toric manifold $M^{2 n}$ can be recorded concisely as a triple $\left(P^{n}, \lambda, M^{2 n}\right)$. Let $K$ be the simplicial complex dual to the boundary of the polytope $P^{n}$. The moment-angle manifold $Z\left(K ;\left(D^{2}, S^{1}\right)\right)$ is a subcomplex of the product of two-discs

$$
Z\left(K ;\left(D^{2}, S^{1}\right)\right) \subseteq\left(D^{2}\right)^{m} \subset \mathbb{C}^{m} .
$$

As such, it has a natural action of the real $m$-torus $T^{m}$. If $\lambda$ satisfies the condition following (3), then the kernel of $\lambda$, a subgroup $T^{m-n} \subset T^{m}$, acts freely on $Z\left(K ;\left(D^{2}, S^{1}\right)\right)$ and the quotient is homeomorphic to $M^{2 n}[\mathbf{1 2}$, p. 434], and [9, p. 86]. The action of $T^{n}$ on $M^{2 n}$, which yields $P^{n}$ as orbit space, is that given by the action of the quotient $T^{m} / T^{m-n}$ on $Z\left(K ;\left(D^{2}, S^{1}\right)\right) / T^{m-n}$. 
The freeness of the action of $T^{m-n}$ on $Z\left(K ;\left(D^{2}, S^{1}\right)\right)$ implies a homotopy equivalence of Borel spaces

$$
E T^{m} \times_{T^{m}} Z\left(K ;\left(D^{2}, S^{1}\right)\right) \longrightarrow E T^{n} \times_{T^{n}} M^{2 n} .
$$

Moreover, for any simplicial complex $K$, there is an equivalence [9]

$$
E T^{m} \times_{T^{m}} Z\left(K ;\left(D^{2}, S^{1}\right)\right) \cong Z\left(K ;\left(\mathbb{C} P^{\infty}, *\right)\right),
$$

where the right-hand side is a polyhedral product which is a subcomplex of the product space $\left(\mathbb{C} P^{\infty}\right)^{m}$. These spaces are called (loosely) the Davis-Januszkiewicz spaces associated to the simplicial complex $K$ and are denoted by the symbol $\mathcal{D} \mathcal{J}(K)$. Also, the cohomology ring $H^{*}(\mathcal{D} \mathcal{J}(K) ; \mathbb{Z})$ is the Stanley-Reisner ring of the simplicial complex $K$. The Serre spectral sequence of the fibration

$$
M^{2 n} \longrightarrow E T^{n} \times_{T^{n}} M^{2 n} \stackrel{p}{\longrightarrow} B T^{n}
$$

yields an entirely topological computation of the ring $H^{*}(M ; \mathbb{Z})$. Known as the DavisJanuszkiewicz theorem, it generalizes the Danilov-Jurkewicz theorem for projective non-singular toric varieties, [12]. Applied to the manifolds $M(J)$, it gives Theorem 4.1.

Given $\left(P^{n}, \lambda, M^{2 n}\right)$, let $\lambda(J)$ be the matrix defined by Figure 1. Choosing a standard basis and following [9], the kernel of $\lambda$, as a sub-torus of $T^{m}$, is specified by an $m \times(m-n)$-matrix $S=\left(s_{i j}\right)$. Explicitly, it is given by

$$
\text { ker } \lambda=\left\{\left(e^{2 \pi i\left(s_{11} \phi_{1}+\cdots+s_{1(m-n)} \phi_{m-n}\right)}, \ldots, e^{2 \pi i\left(s_{m 1} \phi_{1}+\cdots+s_{m(m-n)} \phi_{m-n}\right)}\right) \in T^{m}\right\} \text {, }
$$

where $\phi_{i} \in \mathbb{R}, i=1,2, \ldots, m-n$. The form of the matrix in Figure 1 reveals the kernel of $\lambda(J)$ to be specified by the $d(J) \times(m-n)$-matrix $S(J)=\left(s_{l k}^{J}\right)$, where

$$
s_{l k}^{J}= \begin{cases}s_{1 k}, & \text { if } 1 \leqslant l \leqslant j_{1}, \\ s_{2 k}, & \text { if } j_{1}+1 \leqslant l \leqslant j_{1}+j_{2}, \\ s_{3 k}, & \text { if } j_{1}+j_{2}+1 \leqslant l \leqslant j_{1}+j_{2}+j_{3}, \\ \vdots & \\ s_{m k}, & \text { if } j_{1}+j_{2}+\cdots+j_{m-1}+1 \leqslant l \leqslant j_{1}+j_{2}+j_{3}+\cdots+j_{m}=d(J) .\end{cases}
$$

Notice that this description makes explicit the isomorphism: $\operatorname{ker} \lambda(J) \cong \operatorname{ker} \lambda \cong T^{m-n}$.

The action of $\operatorname{ker} \lambda(J)$ on both $Z\left(K ;\left(\underline{D}^{2 J}, \underline{S}^{2 J-1}\right)\right)$ and $\left.Z\left(K(J) ;\left(D^{2}, S^{1}\right)\right)\right)$ is via the inclusions

$$
\operatorname{ker} \lambda(J) \subset T^{m} \subset T^{d(J)},
$$

where the first inclusion is determined by the matrix $S$ and (19); the second is determined by (13). Now, $\operatorname{ker} \lambda(J)$ acts freely on $Z\left(K(J) ;\left(D^{2}, S^{1}\right)\right)$ by Theorem 3.2 and hence on $Z\left(K ;\left(\underline{D}^{2 J}, \underline{S}^{2 J-1}\right)\right)$ by Theorem 7.5 , which implies also the result following.

Lemma 9.1. There is a homeomorphism of orbit spaces

$$
\left.Z\left(K(J) ;\left(D^{2}, S^{1}\right)\right)\right) / \operatorname{ker} \lambda(J) \longrightarrow Z\left(K ;\left(\underline{D}^{2 J}, \underline{S}^{2 J-1}\right)\right) / \operatorname{ker} \lambda(J) .
$$

Remark. The space on the left acquires a differentiable structure from the general topological theory of toric manifolds, $[\mathbf{1 2}, \mathbf{8}]$. The homeomorphism of Lemma 9.1 imposes then a differentiable structure on the space on the right. 
Recall now that Theorem 3.2 and the discussion at the beginning of this section imply that $M(J)$ is diffeomorphic to $\left.Z\left(K(J) ;\left(D^{2}, S^{1}\right)\right)\right) /$ ker $\lambda(J)$. The main theorem follows from Lemma 9.1.

Theorem 9.2. There is a diffeomorphism

$$
M(J) \longrightarrow Z\left(K ;\left(\underline{D}^{2 J}, \underline{S}^{2 J-1}\right)\right) / \operatorname{ker} \lambda(J) .
$$

\section{The analogue of the Davis-Januszkiewicz space}

The fibration (18) is used in the standard theory to exhibit $H^{*}\left(M^{2 n} ; \mathbb{Z}\right)$ as a quotient of the cohomology of the Davis-Januszkiewicz space. In its various guises, this space is

$$
\mathcal{D} \mathcal{J}(K):=E T^{n} \times_{T^{n}} M^{2 n} \simeq E T^{m} \times_{T^{m}} Z\left(K ;\left(D^{2}, S^{1}\right)\right) \simeq Z\left(K ;\left(\mathbb{C} P^{\infty}, *\right)\right) .
$$

The recognition of $M(J)$ as the quotient $Z\left(K(J) ;\left(D^{2}, S^{1}\right)\right) / \operatorname{ker} \lambda(J)$ yields the cohomology calculation in Theorem 4.1. To get the more concise calculation afforded by Theorem 4.2, the description of $M(J)$ given by Theorem 9.2 is used instead. Therefore, an appropriate analogue of the space $\mathcal{D} \mathcal{J}(K)$ is needed.

Define a family of $\mathrm{CW}$ pairs by

$$
\left(\underline{\mathbb{C} P}^{\infty}, \underline{\mathbb{C}}^{J-1}\right):=\left\{\left(\mathbb{C} P^{\infty}, \mathbb{C} P^{j_{1}-1}\right), \ldots,\left(\mathbb{C} P^{\infty}, \mathbb{C} P^{j_{m}-1}\right)\right\}
$$

and consider the polyhedral product

$$
Z\left(K ;\left(\underline{\mathbb{C} P}^{\infty}, \underline{\mathbb{C}}^{J-1}\right)\right) \subseteq\left(\mathbb{C} P^{\infty}\right)^{m}=B T^{m} .
$$

Theorem 10.1. There is a homotopy equivalence

$$
E T^{m} \times_{T^{m}} Z\left(K ;\left(\underline{D}^{2 J}, \underline{S}^{2 J-1}\right)\right) \stackrel{\alpha}{\longrightarrow} Z\left(K ;\left(\underline{\mathbb{C}}^{\infty}, \underline{\mathbb{C}}^{J-1}\right)\right)
$$

making the following diagram commute:

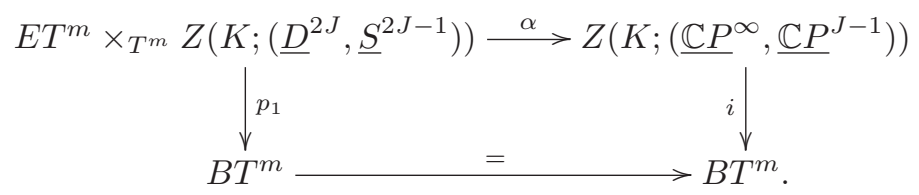

Proof. For the pair $(\underline{X}, \underline{A})=\left(\underline{D}^{2 J}, \underline{S}^{2 J-1}\right)$, consider $D(\sigma)$ as in (9). The action of $T^{m}$ leaves $D(\sigma)$ invariant, so

$$
E T^{m} \times_{T^{m}} Z\left(K ;\left(\underline{D}^{2 J}, \underline{S}^{2 J-1}\right)\right)=E T^{m} \times_{T^{m}}\left(\cup_{\sigma \in K} D(\sigma)\right)=\bigcup_{\sigma \in K} E T^{m} \times_{T^{m}} D(\sigma) .
$$

The torus $T^{m}$ acts diagonally, so it suffices to observe that, as a pair,

$$
\left(E T^{1} \times_{T^{1}} D^{2 j_{k}}, E T^{1} \times_{T^{1}} S^{2 j_{k}-1}\right) \simeq\left(\mathbb{C} P^{\infty}, \mathbb{C} P^{j_{k}-1}\right) .
$$

This follows by an argument based on the fact that the left-hand side is a disc-sphere bundle pair over $\mathbb{C} P^{\infty}$.

$D^{2 j_{k}}$ is contractible and $T^{1}$ acts freely on $S^{2 j_{k}-1}$. Therefore, the Borel construction converts $D(\sigma)$ for the family of pairs $\left(\underline{D}^{2 J}, \underline{S}^{2 J-1}\right)$ into $D(\sigma)$ for the family of pairs $\left(\underline{\mathbb{C} P}^{\infty}, \mathbb{C} P^{J-1}\right)$. Moreover, the map $\alpha$ is constructed as a factorization of $p_{1}$, so the diagram does commute. 
Remark 10.2. It is necessary to record certain standard facts about cell decompositions and their implications for the polyhedral product complexes $Z\left(K ;\left(\mathbb{C P}^{\infty}\right.\right.$, $\left.\left.\underline{\mathbb{C} P^{J-1}}\right)\right)$. The classical example of $\mathrm{C}$. Dowker [14] shows that some care is needed.

Let $J=\left(j_{1}, j_{2}, \ldots, j_{m}\right)$ be as above, and fix $N>j_{i}-1, i=1,2, \ldots, m$. For $\sigma \in K$, consider the space

$$
D^{N}(\sigma)=\prod_{i=1}^{m} W_{i}, \quad \text { where } \quad W_{i}= \begin{cases}\mathbb{C} P^{N} & \text { if } \quad i \in \sigma \\ \mathbb{C} P^{j_{i}-1} & \text { if } \quad i \in[m]-\sigma .\end{cases}
$$

The compact spaces $\mathbb{C} P^{N}$ and $\mathbb{C} P^{j_{i}-1}, i=1,2, \ldots, m$ are each assumed to be given the $\mathrm{CW}$ decomposition with one cell in each even dimension up to the top. This induces a cell decomposition of the product $D^{N}(\sigma)$, with each cell homeomorphic to a product of cells of even dimension, each in one of the spaces $W_{i}$. The compactness implies that the product topology and compactly generated topology agree.

Consider now the spaces $D(\sigma)$, from $(9)$, in $Z\left(K ;\left(\underline{\mathbb{C}}^{\infty}, \underline{\mathbb{C}}^{J-1}\right)\right)$ :

$$
D(\sigma)=\prod_{i=1}^{m} W_{i}, \quad \text { where } \quad W_{i}=\left\{\begin{array}{lll}
\mathbb{C} P^{\infty} & \text { if } \quad i \in \sigma \\
\mathbb{C} P^{j_{i}-1} & \text { if } \quad i \in[m]-\sigma .
\end{array}\right.
$$

The spaces $D(\sigma)$ are each a colimit, over increasing $N$, of the spaces $D^{N}(\sigma)$. The colimit is via compatible inclusions, and so each space $D(\sigma)$ inherits a CW structure with cells in even dimension. Finally,

$$
Z\left(K ;\left(\underline{\mathbb{C} P}^{\infty}, \underline{\mathbb{C} P}^{J-1}\right)\right)=\bigcup_{\sigma \in K} D(\sigma)
$$

is a finite colimit of spaces that have compatible cell structures on intersections, and so inherits a cell structure with cells concentrated in even dimension.

From these considerations follows the next lemma.

Lemma 10.3. The inclusion of the subcomplex $Z\left(K ;\left(\underline{\mathbb{C}}^{\infty}, \underline{\mathbb{C} P^{J-1}}\right)\right) \subseteq B T^{m}$ induces a surjective map

$$
H^{*}\left(B T^{m} ; \mathbb{Z}\right) \longrightarrow H^{*}\left(Z\left(K ;\left(\underline{\mathbb{C} P}^{\infty}, \underline{\mathbb{C} P^{J-1}}\right)\right) ; \mathbb{Z}\right) .
$$

Let $I_{K}^{J}$ be the ideal in $\mathbb{Z}\left[v_{1}, v_{2}, \ldots, v_{m}\right]$ described in Theorem 4.2. It is generated by all monomials $v_{i_{1}}^{j_{i_{1}}} v_{i_{2}}^{j_{i_{2}}} \cdots v_{i_{k}}^{j_{i_{k}}}$ corresponding to minimal non-simplices $\left\{v_{i_{1}}, v_{i_{2}}, \ldots\right.$, $\left.v_{i_{k}}\right\}$ of $K$.

Definition 10.4. Let $K$ be a simplicial complex on $m$ vertices, and let $J=\left(j_{1}, j_{2}, \ldots\right.$, $\left.j_{m}\right)$, be a sequence of positive integers. The ring $\mathbb{Z}\left[v_{1}, v_{2}, \ldots, v_{m}\right] / I_{K}^{J}$ is called the $J$ weighted Stanley-Reisner ring of $K$ and is denoted by the symbol $S R^{J}(K)$. The polyhedral product $Z\left(K ;\left(\mathbb{C} P^{\infty}, \mathbb{C} P^{J-1}\right)\right)$ is called the $J$-weighted Davis-Januszkiewicz space and is denoted by $\mathcal{D} \mathcal{J}^{J}(K)$.

Theorem 10.5. There is an isomorphism of rings

$$
H^{*}\left(Z\left(K ;\left(\underline{\mathbb{C} P}^{\infty}, \underline{\mathbb{C}}^{J-1}\right)\right) ; \mathbb{Z}\right) \longrightarrow \mathbb{Z}\left[v_{1}, v_{2}, \ldots, v_{m}\right] / I_{K}^{J} .
$$


Proof. Since $Z\left(K ;\left(\underline{\mathbb{C}}^{\infty}, \underline{\mathbb{C} P^{J-1}}\right)\right)$ is a cellular subcomplex of $\left(\mathbb{C} P^{\infty}\right)^{m}$, having cells in even degree only, the kernel of the surjective map

$$
\left.H^{*}\left(\mathbb{C} P^{\infty}\right)^{m} ; \mathbb{Z}\right) \longrightarrow H^{*}\left(Z\left(K ;\left(\underline{\mathbb{C} P}^{\infty}, \underline{\mathbb{C}}^{J-1}\right)\right) ; \mathbb{Z}\right)
$$

is determined by an argument entirely analogous to that of [8, Proposition 4.3.1].

Remark 10.6. This result has been extended by the authors in [4] to realize geometrically a large class of monomial ideal rings using simplicial complexes. The construction itself generalizes to realize all monomial ideal rings.

\section{Generalized moment-angle complexes and the cohomology of $M(J)$}

The computation of $H^{*}\left(M^{2 n} ; \mathbb{Z}\right)$ in [12] is generalized to recover Theorem 4.2 directly from the results of the previous section. Traditionally, $([\mathbf{1 2}, \mathbf{9}])$, the canonical diagram of fibrations

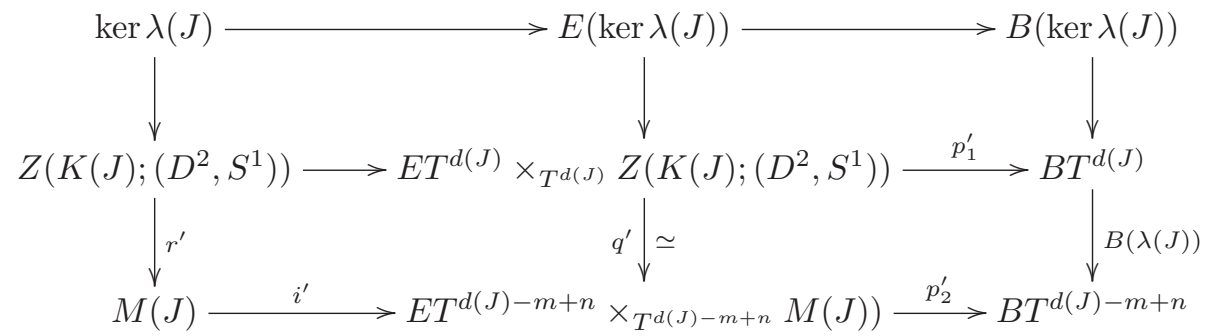

is used, in conjunction with the Serre spectral sequence of the fibration in the bottom row, to obtain the standard description of $H^{*}\left(M^{2 n} ; \mathbb{Z}\right)$ given by Theorem 4.1.

The more condensed calculation in Theorem 4.2 is obtained by considering instead the homotopy commutative diagram of fibrations

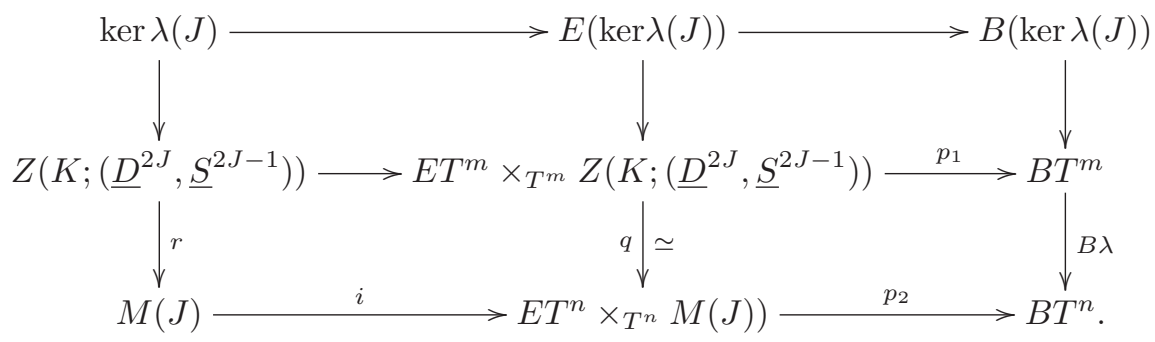

In the diagram, $r$ is the map given by Theorem 9.2 and the insertion of the map $B \lambda$ is possible by (19) and the comment following it. The equivalence $q$ is a consequence of the splitting $T^{m} \cong \operatorname{ker} \lambda(J) \times T^{n}$ as topological groups and the fact that $\operatorname{ker} \lambda(J)$ acts freely on $Z\left(K ;\left(\underline{D}^{2 J}, \underline{S}^{2 J-1}\right)\right)$.

Theorem 10.1 allows the replacement, up to homotopy, of the fibration along the bottom row of the diagram with

$$
M(J) \longrightarrow Z\left(K ;\left(\underline{\mathbb{C}}^{\infty}, \underline{\mathbb{C}}^{J-1}\right)\right) \stackrel{p}{\longrightarrow} B T^{n} .
$$


These observations allow for an alternative approach to the calculation of Theorem 4.2 .

Theorem 11.1. There is an isomorphism of rings

$$
H^{*}(M(J) ; \mathbb{Z}) \longrightarrow H^{*}\left(Z\left(K ;\left(\underline{\mathbb{C}}^{\infty}, \underline{\mathbb{C}}^{J-1}\right)\right) ; \mathbb{Z}\right) / L,
$$

where $L$ is the two-sided ideal generated by the image of the map $p^{*}$.

Proof. In the Serre spectral sequence associated with (23), all groups are concentrated in even degree. This is true for $H^{*}(M(J) ; \mathbb{Z})$ because $M(J)$ is a toric manifold by Theorem 3.2 and, for $H^{*}\left(Z\left(K ;\left(\underline{\mathbb{C}}^{\infty}, \mathbb{C} P^{J-1}\right)\right) ; \mathbb{Z}\right)$, by Theorem 10.5. The spectral sequence collapses. The $E_{2}$-term is given by

$$
H^{*}(M(J)) \otimes H^{*}\left(B T^{n}\right) .
$$

It follows that $H^{*}(M(J))$ is the quotient of $H^{*}\left(Z\left(K ;\left(\underline{\mathbb{C} P}^{\infty}, \underline{\mathbb{C}}^{J-1}\right)\right)\right)$ by the twosided ideal generated by the image of $p^{*}$.

It remains to identify the ideal $L$ in Theorem 11.1. With reference to the righthand bottom square in diagram (22), the map $p^{*}$ is the composition $\left(\alpha^{-1}\right)^{*} \circ\left(p_{1}^{*} \circ s^{*}\right)$, where the map $\alpha$ is the equivalence of Theorem 10.1. Therefore, the image of $p^{*}$ is the image of the composition

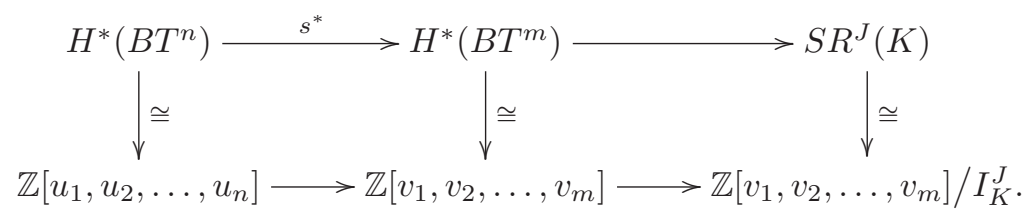

This is specified by (19) and generates the ideal $L_{M}$ of Theorem 4.2, determined by the rows of the original matrix $\lambda$. Therefore, $L=L_{M}$ and Theorem 11.1 becomes

$$
H^{*}(M(J) ; \mathbb{Z}) \cong \mathbb{Z}\left[v_{1}, v_{2}, \ldots, v_{m}\right] /\left(I_{K}^{J}+L_{M}\right),
$$

recovering Theorem 4.2 completely from the topological point of view of generalized moment-angle complexes.

\section{Nests}

Let $J=\left(j_{1}, j_{2} \ldots, j_{m}\right)$ and $L=\left(l_{1}, l_{2}, \ldots, l_{m}\right)$ be sequences of positive integers. The direct product ordering on such sequences is given by $J<L$ if $j_{i} \leqslant l_{i}$ for all $i$ and, for at least one $k \in\{1,2, \ldots, m\}, j_{k}<l_{k}$. If $J<L$, the inclusions $D^{2 j_{i}} \subseteq D^{2 l_{i}}$ induce an equivariant embedding

$$
Z\left(K ;\left(\underline{D}^{2 J}, \underline{S}^{2 J-1}\right)\right) \subset Z\left(K ;\left(\underline{D}^{2 L}, \underline{S}^{2 L-1}\right)\right)
$$

and, consequently, an embedding $\zeta: M(J) \rightarrow M(L)$. The next proposition follows easily. 
Proposition 12.1. For $J<L$, the normal bundle of the embedding $\zeta$ is

$$
\bigoplus_{i=1}^{m}\left(l_{i}-j_{i}\right) \alpha_{i}
$$

where $\alpha_{i}$ is the line bundle over $M(J)$ with first Chern class $c_{i}\left(\alpha_{i}\right)=v_{i}$, the class from the cohomology description (24). Moreover, the induced map

$$
i^{*}: H^{k}(M(L) ; \mathbb{Z}) \longrightarrow H^{k}(M(J) ; \mathbb{Z})
$$

is an epimorphism that is an isomorphism for $k=2$.

Proof. Diagram (22) implies that the diagram below commutes up to homotopy. (Notice here that the rows are not fibrations up to homotopy.)

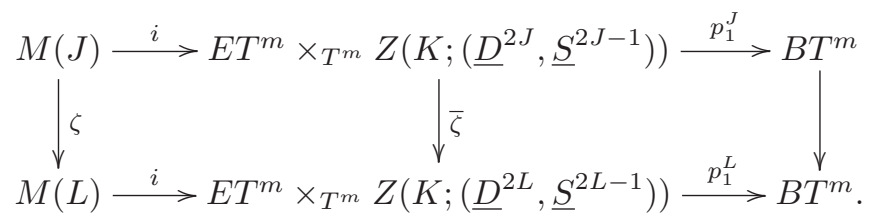

Theorems 10.1 and 10.5 imply that $\bar{\zeta}^{*}$ is onto, and the Serre spectral sequence part of the proof of Theorem 11.1 shows that $i^{*}$ is onto. This implies that $\zeta^{*}$ is onto too. The first part of the proposition follows from the fact that each canonical bundle $L_{i}$ over $B T^{m}$ pulls back to the bundle $l_{i} \alpha_{i}$ over $M(L)$ and to $j_{i} \alpha_{i}$ over $M(J)$.

A "nest" of toric manifolds is constructed below from a given toric manifold triple $\left(P^{n}, M^{2 n}, \lambda\right)$, an initial sequence $J_{0}=(1,1, \ldots, 1)$ with $m$ entries corresponding to the number of facets of $P^{2 n}$ and a sequence $J_{0}<J_{1}<J_{2}<\cdots$ with the property that $d\left(J_{i+1}\right)=d\left(J_{i}\right)+1$. (The symbol $d(J)$ is defined at the end of Section 2).

Proposition 12.2. Given a toric manifold $\left(P^{n}, M^{2 n}, \lambda\right)$, and a sequence $J_{0}<J_{1}<$ $J_{2}<\cdots$ as above, there is a nest of toric manifolds

$$
M^{2 n}=M\left(J_{0}\right) \subset M\left(J_{1}\right) \subset \cdots \subset M\left(J_{k}\right) \subset \ldots,
$$

where $d\left(J_{i}\right)=m+i$, the dimension of $M\left(J_{i}\right)$ is $2 n+2 i$ and $M\left(J_{i}\right) \subset M\left(J_{i+1}\right)$ is a codimension-two embedding. Furthermore, there is a sequence of epimorphisms

$$
\cdots \longrightarrow H^{k}\left(M\left(J_{i}\right) ; \mathbb{Z}\right) \longrightarrow H^{k}\left(M\left(J_{i-1}\right) ; \mathbb{Z}\right) \longrightarrow \cdots \longrightarrow H^{k}\left(M^{2 n} ; \mathbb{Z}\right)
$$

which are isomorphisms for $k=2$.

Problem: Beginning with a toric manifold $\left(P^{n}, M^{2 n}, \lambda\right)$, find invariants, possibly in terms of $\lambda$, that will detect diffeomorphic (homotopic) manifolds in nests corresponding to different sequences $J_{0}<J_{1}<J_{2}<\cdots$.

\section{References}

[1] A. Bahri, M. Bendersky, F.R. Cohen, and S. Gitler, Decompositions of the polyhedral product functor with applications to moment-angle complexes and related spaces, Proc. Natl. Acad. Sci. USA 106 (2009), 12241-12244. 
[2] A. Bahri, M. Bendersky, F. Cohen and S. Gitler, The polyhedral product functor: A method of computation for moment-angle complexes, arrangements and related spaces, Adv. Math. 225 (2010), 1634-1668.

[3] A. Bahri, M. Bendersky, F. Cohen, and S. Gitler, Cup-products for the polyhedral product functor, Math. Proc. Cambridge Philos. Soc. 153 (2012), 457-469, available at http://arxiv.org/abs/1001.3372.

[4] A. Bahri, M. Bendersky, F. Cohen, and S. Gitler, The geometric realization of monomial ideal rings and a theorem of Trevisan, Homology, Homotopy Appl. 15 (2013), 1-7.

[5] A. Bahri, M. Bendersky, F. Cohen, and S. Gitler, A generalization of the DavisJanuszkiewicz construction and applications to toric manifolds and iterated polyhedral products, available at http://arxiv.org/abs/1311.4256.

[6] F. Bosio and L. Meersseman, Real quadrics in $\mathbb{C}^{n}$, complex manifolds, and convex polytopes, Acta Math. 197 (2006), 53-127.

[7] V. Buchstaber and T. Panov, Torus actions, combinatorial topology and homological algebra, Uspekhi Mat. Nauk 55 (2000), no. 5, 3-106 (Russian). Russian Math. Surveys 55 (2000), no. 5, 825-921 (English translation), arXiv:math.AT/0010073.

[8] V. Buchstaber and T. Panov, Toric Topology, AMS Mathematical Surveys and Monographs, 204 (2015).

[9] V. Buchstaber and T. Panov, Torus actions and their applications in topology and combinatorics, AMS University Lecture Series, 24, (2002).

[10] S. Choi and H. Park, Wedge operations and torus symmetries, to appear in Tohoku Math. J. Available at http://arxiv.org/abs/1305.0136.

[11] S. Choi, S. Park, and D.Y. Suh, Topological classification of quasitoric manifolds with second Betti number 2, Pacific J. Math. 256 (2012), no. 1, 19-49.

[12] M. Davis, and T. Januszkiewicz, Convex polytopes, Coxeter orbifolds, and torus actions, Duke Math. J. 62 (1991), no. 2, 417-451.

[13] G. Denham and A. Suciu, Moment-angle complexes, monomial ideals and Massey products, Pure Appl. Math. Q. 3 (2007), no. 1, 25-60.

[14] C. Dowker, Topology of metric complexes, Amer. J. Math. 74 (1952), 555-577.

[15] N. Erokhovets, The Buchstaber invariant of simple polytopes, Uspekhi Mat. Nauk 63 (2008), no. 5, 187-188 (Russian); Russ. Math. Surv. 63 (2008), no. 5, 962-964 (English translation).

[16] G. Ewald, Spherical complexes and nonprojective toric varieties, Discrete Comput. Geom. 1 (1986), 115-122.

[17] S. Gitler and S. López de Medrano, Intersections of quadrics, moment-angle manifolds, and connected sums, Geom. Topol. 17 (2013), no. 3, 1497-1534.

[18] J. Grbic and S. Theriault, The homotopy type of the polyhedral product for shifted complexes, Adv. Math. 245 (2013), 690-715.

[19] J. Grbic, T. Panov, S. Theriault, and J. Wu, Homotopy types of moment-angle complexes for flag complexes, available at http://arxiv.org/abs/1211.0873. 
[20] S. López de Medrano, Topology of the intersection of quadrics in $\mathbb{R}^{n}$, Algebraic Topology, Arcata California, 1986, Lecture Notes in Math. 1370 SpringerVerlag (1989), 280-292.

[21] S. López de Medrano, The space of Siegel leaves of a holomorphic vector field, Holomorphic Dynamics, Mexico, 1986, Lecture Notes in Math. 1345 SpringerVerlag (1988), 233-245.

[22] J.S. Provan and L.J. Billera, Decompositions of simplicial complexes related to diameters of convex polyhedra, Math. Oper. Res. 5 (1980), 576-594.

[23] A. Suciu, The rational homology of real toric manifolds, available online at http://arxiv.org/abs/1302.2342.

[24] Y. Ustinovsky, Doubling operation for polytopes and torus actions, Uspekhi Mat. Nauk 64 (2009), no. 5, 181-182 (Russian); Russ. Math. Surv. 64 (2009), no. 5, 952-954 (English translation).

[25] Y. Ustinovsky, Toral rank conjecture for moment-angle complexes, Mat. Zametki 90 (2011), no. 2, 300-305 (Russian); Math. Notes 90 (2011), no. 2, 279-283.

A. Bahri bahri@rider.edu

Department of Mathematics, Rider University, Lawrenceville, NJ 08648, USA

M. Bendersky mbenders@hunter.cuny.edu

Department of Mathematics, CUNY, East 695 Park Avenue, New York, NY 10065, USA

F. R. Cohen cohf@math.rochester.edu

Department of Mathematics, University of Rochester, Rochester, NY 14625, USA

S. Gitler (Deceased)

El Colegio Nacional, Gonzalez Obregon 24 C, Centro Historico, Mexico City, Mexico 